$1-31-2017$

\title{
A Critical Discussion of Patient Engagement in Research
}

Andrea Elaine Bombak

Heather M. Hanson

Follow this and additional works at: https://aah.org/jpcrr

Part of the Health Services Research Commons, Medical Education Commons, and the Public Health Education and Promotion Commons

\section{Recommended Citation}

Bombak AE, Hanson HM. A critical discussion of patient engagement in research. J Patient Cent Res Rev. 2017;4:39-41. doi: 10.17294/2330-0698.1273

Published quarterly by Midwest-based health system Advocate Aurora Health and indexed in PubMed Central, the Journal of Patient-Centered Research and Reviews (JPCRR) is an open access, peer-reviewed medical journal focused on disseminating scholarly works devoted to improving patient-centered care practices, health outcomes, and the patient experience. 


\title{
A Critical Discussion of Patient Engagement in Research
}

\author{
Andrea Elaine Bombak, ${ }^{1} \mathrm{PhD}$, Heather M. Hanson, $\mathrm{PhD}^{2,3}$ \\ ${ }^{1}$ Division of Community Health, Central Michigan University, Mt. Pleasant, MI \\ ${ }^{2}$ Department of Community Health Sciences, Cumming School of Medicine, University of Calgary, Calgary, Alberta, Canada \\ ${ }^{3}$ Seniors Health Strategic Clinical Network, Alberta Health Services, Calgary, Alberta, Canada
}

\begin{abstract}
Patients are increasingly expected to take a more involved role in research. Funding for some projects now requires incorporating patients' viewpoints or involvement in research processes. While intended to achieve commendable goals, it is important to critically assess the means used to achieve these aims. Presently, there may be issues of valuing only certain epistemologies, failing to evaluate existing programs and their impacts, marginalizing less "engaged" patients, and promoting only tokenism. These are areas that require exploration and reflection before assuming that patient engagement approaches are sufficient or the only means of incorporating patient perspectives into research. (J Patient Cent Res Rev. 2017;4:39-41.)
\end{abstract}

Keywords patient engagement; public involvement; patient activations; patient-centered outcomes research

Patient engagement is increasingly sought in research settings and has been incorporated into funding agendas and institutional missions. ${ }^{1,2}$ Patient engagement in research is considered an avenue for producing democratic, patient-centered health services. The Canadian Institutes of Health Research (CIHR) defines patient engagement as "[what] occurs when patients meaningfully and actively collaborate in the governance, priority setting, and conduct of research, as well as in summarizing, distributing, sharing, and applying its resulting knowledge."'

Patient engagement in research was developed to meet laudable goals; however, as social scientists involved in clinical research teams, we have concerns regarding the lack of critical scholarship and wholesale investment into narrowly defined methods of conducting patient engagement. It is necessary to seriously reflect on approaches designed to incorporate patient involvement and insights into research to determine whether they are inadvertently causing unanticipated problems.

\section{Patient Engagement in Research}

Researchers seek to engage patients in research through a variety of methods. Most often these approaches

Correspondence: Andrea Elaine Bombak, PhD,

Central Michigan University, 2238 Health Professions Building,

Mt. Pleasant, MI, 48859, T: 989-774-3607,

Email: bomba1a@cmich.edu involve less-intensive inclusion of patients through interviews, surveys or focus groups during the research process. ${ }^{3,4}$ Approaches involving more intensive forms of patient engagement are becoming increasingly popular, and some international funding agencies now require patient engagement in research.

More intensive forms of engagement can include training patient-researchers through a specific curriculum. ${ }^{5,6}$ These curricula focus on bridging patient and research cultures to produce patient-researchers capable of joining research teams. This derived from concerns that patients need training to feel validated by scientific terminology and processes. ${ }^{7}$ However, training patient-researchers in this manner — by teaching patient-researchers to conduct research according to prescribed, validated academic parameters suggests a form of acculturation. Importantly, patients do not constitute a monolithic culture exclusive from researchers; patients may have preexisting research expertise. Intensive forms of patient engagement in research may hinder involvement of patients without abundant time and interest to invest. This may explain, for example, CIHR's recent desire to move beyond "the usual suspects," 8 i.e. the same patients already involved in research endeavors.

Patient-researcher training programs may privilege certain forms of knowledge, especially observable or quantitative phenomena. This focus on particular forms 
of data can silence and alienate patients whose worldview relies on other forms of information, including instinct, embodiment and intuition. Ultimately, this exclusion may foreclose valuable insights from entering research, practice and policy. As prescribed ways of doing patient engagement gain credence, it is essential to remember the contributions of critical qualitative and communitybased researchers who have been learning from patients and valuing their experiential knowledge for decades.

Patient perspectives on these new roles are critical to consider. Studies indicate some patients have internalized a moral imperative of "patient engagement," including preventive behaviors and research participation., Taken to the extreme, the role of an "engaged" patient would appear to be a rational, well-informed actor who eases system and practice burden by making clinically sanctioned decisions and contributing to research. While some patients embrace these opportunities, lesseager patients or those unable to be intensely involved may feel shamed. Potentially interested patients may be marginalized if the only avenue for engagement is especially time- or resource-intensive or operates from only specific worldviews that patients may find alienating or unappealing. Patients who decline engagement opportunities may be labeled "disinterested" for not contributing to scholarship.

Relatedly, attempts to engage public involvement in research have raised concerns over tokenism, a superficial and disingenuous display of inclusion in which a small number of participants, who are often little involved in the actual research process, are viewed as representative of a far larger and diverse patient group. ${ }^{3,710}$ Furthermore, the structures in place for patient participation can be confusing, disorganized and limited. ${ }^{10}$ Systematic reviews on patient or end-user/consumer involvement in research indicate that patient engagement is feasible in many settings; however, drawbacks include patients' excessive time commitment, inappropriate widening of the scope of research and potential tokenism. ${ }^{3}$ The reviewers found that patient engagement could reduce attrition, improve enrollment and help produce more comprehensible and relevant materials for patients. ${ }^{3,11}$ However, definitions and theoretical underpinnings of what constitutes patient engagement are lacking, and inadequate measures and reporting affect evaluation of impact. ${ }^{4}$ Moreover, little relevant evidence produced by these efforts has been taken up in policy. ${ }^{12}$
More evaluative work is needed to allow a rigorous assessment of patient engagement's value to research and patient preferences for research engagement. Reflective and critical perspectives of patient engagement are necessary, as much scholarly output in this area is confined to work undertaken with oversight of patient advisory boards, performed by patient-centered outcomes research institutes or funded through related initiatives. While this approach has produced many valuable articles, ${ }^{3,4,12}$ research into patient engagement also must be conducted by those scholars who may be less personally invested in patient engagement. Participants in studies examining the patient engagement process may feel constrained in their responses when these studies are conducted by researchers who are notably pro-public engagement. ${ }^{13}$ A more productive and thoughtful understanding of patient engagement requires research from scholars and participants with a wide range of perspectives.

At this stage in determining the ideal role for patients in research, funding and judgments of quality should not be based on specific models of patient engagement but include multiple forms of patient engagement and knowledge generation. This is especially true given patient engagement's lack of clear definition and methodologies, and patients' and providers' divided investment. Further research on patient preferences would clarify the research roles patients actually desire, rather than furthering the notion that more intensive "engagement" among a very select few "activated" patients is necessarily always preferable.

\section{Researcher and Provider Perspectives on Patient Engagement}

Many authors recognize the potential resistance of providers and researchers to patient engagement. Health researchers from the United Kingdom expressed appreciation for the worth of public research involvement, although reservations were expressed involving the extent of public engagement, deprofessionalization (given the public's presumed lack of expertise) and relevance to clinician-researchers. ${ }^{13}$ Some participants conceptualized engagement as akin to dissemination, which was considered a tool to enhance compliance. This suggests patient engagement is still viewed as a biomedically led technique for ensuring adherence. Concerns also were expressed that political salience of public involvement would produce disingenuous lip service. ${ }^{13}$ 
These reservations reinforce the need for caution in viewing patient engagement as the only valid method of seeking to give all patients opportunities to raise concerns within research. As patient engagement becomes institutionalized and incorporated into funding schemes, it may silence other ways of knowledge acquisition, differing methods of exploration and involvement, and the valid and informative views of patients who may not fit the "engagement" mold.

\section{Conclusions}

Greater data on what patients desire in research opportunities are needed to ensure all patients are accommodated. Clarifying patient understandings of engagement is essential for following through on patient-centered care. Consideration of the extent to which patient engagement efforts impact health service and policy is also necessary to ensure the time and effort of patients, researchers and professionals is expended in the most valuable manner. There are multiple methodologies from numerous disciplines that can (and have been) used to involve patients.

Conducting research that values patients' expertise and priorities is a worthy goal and should not be executed in a tokenistic, rote manner. We must guard against these slippery impulses to value certain forms of knowledge over others by critically evaluating existing approaches, advancing theory and methods, and gathering evidence from patients and other stakeholders rather than assuming that existing approaches, because they are well-intentioned, are necessarily the best.

\section{Patient-Friendly Recap}

- Requiring patient engagement in health research projects is a growing trend among care providers and funding organizations.

- Concerns have been raised regarding the research community's embrace of untested and relatively narrow approaches to engaging patients in study designs.

- The authors briefly reviewed the current state of patient contributions to research and identified possible improvements, such as expanding the types of acceptable patient involvement and avoiding tokenism.

\section{Conflicts of interest}

None.

\section{Funding Sources}

Andrea Bombak received salary support from Alberta Innovates - Health Solutions (Edmonton, Alberta, Canada) through a PRIHS grant (RES0024176).

\section{References}

1. Canadian Institutes of Health Research. Patient engagement. http://www.cihr-irsc.gc.ca/e/45851.html. Accessed Nov. 30, 2016.

2. Patient-Centered Outcomes Research Institute. What we mean by engagement. http://www.pcori.org/funding-opportunities/ what-we-mean-engagement. Accessed Aug. 19, 2016.

3. Domecq JP, Prutsky G, Elraiyah T, et al. Patient engagement in research: a systematic review. BMC Health Serv Res. 2014;14:89. CrossRef

4. Mockford C, Staniszewska S, Griffiths F, Herron-Marx S. The impact of patient and public involvement on UK NHS health care: a systematic review. Int J Qual Health Care. 2012;24:28-38. CrossRef

5. Canadian Depression Research and Intervention Network. Lived experience training sessions. http://cdrin.org/livedexperience/. Accessed Nov. 17, 2015.

6. O'Brien Institute for Public Health, University of Calgary. PACER - patient and community engagement research. https://obrieniph.ucalgary.ca/pacer. Accessed Nov. 17, 2015.

7. Légaré F, Turcotte S, Stacey D, Ratté S, Kryworuchko J, Graham ID. Patients' perceptions of sharing in decisions: a systematic review of interventions to enhance shared decision making in routine clinical practice. Patient. 2012;5(1):1-19. CrossRef

8. Canadian Institutes of Health Research. Patient engagement: collaboration grants (fall 2015 competition). https://www. researchnet-recherchenet.ca/rnr16/vwOpprtntyDtls.do? prog $=2310 \&$ view $=$ currentOpps\&org $=$ CIHR\&type $=$ EXACT \&resultCount $=25 \&$ sort $=$ program \&all $=1 \&$ masterList $=$ true. Accessed Nov. 16, 2015.

9. Renedo A, Marston C. Developing patient-centred care: an ethnographic study of patient perceptions and influence on quality improvement. BMC Health Serv Res. 2015;15:122. CrossRef

10. Minogue V, Girdlestone J. Building capacity for service user and carer involvement in research: the implications and impact of best research for best health. Int J Health Care Qual Assur. 2010;23:422-35. CrossRef

11. Nilsen ES, Myrhaug HT, Johansen M, Oliver S, Oxman AD. Methods of consumer involvement in developing healthcare policy and research, clinical practice guidelines and patient information material. Cochrane Database Syst Rev. 2006;(3):CD004563. CrossRef

12. Stewart RJ, Caird J, Oliver K, Oliver S. Patients' and clinicians' research priorities. Health Expect. 2011;14:439-48. CrossRef

13. Thompson J, Barber R, Ward PR, et al. Health researchers' attitudes towards public involvement in health research. Health Expect. 2009;12:209-20. CrossRef

(C) 2017 Aurora Health Care, Inc. 